Ann. Génét. Sél. anim., I975, 7 (3), 3II-3I5.

NOTE:

\title{
PREMIÈRE ÉTUDE GYTOGÉNÉTIQUE DANS UN CENTRE ITALIEN D'INSÉMINATION ARTIFICIELLE
}

\author{
Annamaria de GIOVANNI, C. P. POPESCU* et G. SUCCI** \\ avec la collaboration technique de Jeannine Boscher* \\ Istituto di Anatomia e Fisiologia degli Animali Domestici, \\ Facoltà di Agraria, \\ Via Celoria 2, Milano (Italie)
}

*U.N.C.E.I.A. et Laboratoire de Génétique factorielle, I. N. R. A., C. N.R.Z., 78350 Jouy en Josas (France)

** Istituto di Zootecnia Generale,

Facoltà di Agraria,

Via Celoria 2, Milano (Italie)

\section{RÉSUMÉ}

Une étude cytogénétique a été entreprise sur 39 taureaux d'un centre d'insémination artificielle en Italie. Aucune anomalie chromosomique de nombre ou de structure n'a été identifiée, mais un animal de race Holstein Friesian a été trouvé porteur d'un chimérisme $\mathrm{XX} / \mathrm{XY}$. I1 présentait $52,3^{8} \mathrm{p}$. roo de cellules femelles, une fertilité réduite par rapport à la moyenne du centre et un sex-ratio modifié en faveur des femelles.

Le perfectionnement, durant la dernière décennie des techniques cytogénétiques, a permis d'examiner un grand nombre d'animaux et de découvrir plusieurs types d'anomalies chromosomiques chez les bovins.

Les anomalies de nombre connues à ce jour se limitent à quelques cas de trisomie impliquant les autosomes (Herzog et HöHn, I968; Herzog et al., I971 ; MORI et al., 1969 ; Dunn et Johnson, 1972) ou les chromosomes sexuels (BASRUR et al., I964 et RIECK et al., 1970). Les anomalies structurales sont, quant à elles, plus nombreuses, puisqu'on connaît déjà plusieurs types de translocations robertsoniennes (Gustavsson, I969; Pollock, I972 ; Brù̀re et Chapman, r973) en tandem (HANSEN, 1969) ou X/autosome (Gustavsson et al., 1968). Elles n'ont généralement pas d'effet phénotypique visible mais peuvent provoquer des perturbations à la méiose qui se tradui- 
sent par des troubles de fertilité. En raison des risques de propagation d'une anomalie chromosomique par l'insémination artificielle actuellement généralisée dans cette espèce, il est indispensable de connaître le caryotype de tous les animaux reproducteurs.

L'objet de cette note est d'effectuer une première enquête cytogénétique dans le centre d'insémination artificielle de Zorlesco (Milano).

L'étude porte sur 39 animaux appartenant à cinq races différentes (tabl. I). Les cultures de sang intégral ont été effectuées selon la technique de DE Grouchy et al. (1964). Les métaphases ayant la meilleure dispersion ont été photographiées avec un dispositif Leitz-Orthomat sur une pellicule Kodak Microfil. Au total, 2 ro photos ont été faites dont 63 ont été utilisées pour obtenir des caryotypes.

TABLEAU I

Répartition par races des animaux étudiés

\begin{tabular}{|c|c|c|}
\hline Race & $\begin{array}{c}\text { Nombre d'animaux } \\
\text { étudiés }\end{array}$ & Pays d'origine \\
\hline Frisonne & $\begin{array}{r}19 \\
9 \\
5\end{array}$ & $\begin{array}{l}\text { Italie } \\
\text { U.S.A. } \\
\text { Canada }\end{array}$ \\
\hline Brune des Alpes & 2 & Italie \\
\hline Piémontaise & 2 & Italie \\
\hline Charolaise & 1 & France \\
\hline Limousine & 1 & France \\
\hline
\end{tabular}

Sur l'ensemble d'animaux étudiés par les méthodes courantes, aucune anomalie chromosomique de nombre ou de structure n'a été observée. Nous avons toutefois identifié un animal porteur d'un chimérisme leucocytaire $60 \mathrm{XX} / 60 \mathrm{XY}$ (fig. I) avec une fréquence de $52,38 \mathrm{p}$. Ioo de cellules femelles sur ${ }^{4} 47$ étudiées. Il s'agit d'un animal de race Frisonne d'origine américaine, vraisemblablement congénère d'un freemartin car déclaré né jumeau. Sa fertilité, exprimée par le taux de non retour à $60-90$ jours, est de $57,8 \mathrm{I}$ p. Ioo et $48,89 \mathrm{p}$. Ioo sur respectivement 365 et 463 inséminations premières, durant les deux dernières années. Elle est inférieure à la fertilité moyenne du centre qui, elle, est de $62 \mathrm{p}$. 100 . Sur $\mathrm{I}_{4} 8$ descendants connus de cet animal on compte 32 mâles et 116 femelles. Le $\chi^{2}$ calculé pour comparer son sex-ratio au rapport $50 / 50$ donne une différence hautement significative au seuil I pour 1 ooo.

Cette absence d'anomalies chromosomiques numériques et structurales pourrait s'expliquer par le fait que la plupart des animaux étudiés appartiennent à la race Frisonne, race dans laquelle les anomalies chromosomiques semblent avoir une fréquence peu élevée. En effet, sur plus d'un millier d'animaux de cette race étudiés aux U.S. A. (Fechнeimer, 1973) en Grande-Bretagne (Pollock, 1974) et en France (Popescu, 1975, en préparation) on n'a trouvé que deux cas de translocations robertsoniennes : une de type 2/4 (Pollock, 1974) et une de type $1 / 29$ (Herschler et FechHeimer, 1966).

Il semble par contre, que le chimérisme chromosomique $\mathrm{XX} / \mathrm{XY}$ ait une fréquence relativement élevée dans tous les rameaux Frisons : 2 p. roo environ de l'ensemble d'animaux mâles étudiés (tabl. 2). 
Le chimérisme chromosomique $\mathrm{XX} / \mathrm{XY}$ caractérise la condition de freemartinisme, phénomène d'intersexualité qui apparaît dans la plupart des naissances gémellaires hétérosexuelles.

Une première explication de ce phénomène a été donnée par LILLIE (1916, 1917) et KelleR et TANDLER (rgI6); elle consisterait en une modification des organes génitaux femelles sous l'influence d'une hormone testiculaire du jumeau mâle à travers les anastomoses placentaires. La découverte par OHNO et al. (1962) du chimérisme $\mathrm{XX} / \mathrm{XY}$ chez les freemartins a fourni la base d'une nouvelle hypothèse. Ainsi Fechheimer (1963) et Bouters et Vandeplassche (I964) pensent que le freemartinisme est provoqué par un transfert des cellules entre les deux jumeaux, le chromosome $\mathrm{Y}$ étant considéré comme la cause majeure de la modification des gonades femelles. D'autres théories ont été formulées depuis, mais aucune n'est actuellement totalement acceptable.

Du point de vue zootechnique le problème du freemartinisme se pose différemment, selon le sexe considéré. Si la femelle freemartin est automatiquement éliminée de la reproduction parce que stérile, le congénère mâle, lui est généralement gardé et utilisé, même en insémination artificielle. A cause de l'absence de malformations phénotypiques, seule une analyse chromosomique peut révéler l'existence du chimérisme chez les mâles nés d'une naissance gémellaire.

Certains auteurs recommandent d'éliminer de la reproduction les mâles congénères des freemartins, soit en raison d'un taux anormalement bas de la testostérone et d'une hypoplasie de la vésicule séminale (SHORT et al., 1969) soit en raison d'une mauvaise fertilité et de la qualité médiocre du sperme (STAFFord, 1972).

Toutefois le sex-ratio dévié en faveur des femelles, trouvée par DunN et al. (1969) et LodjA (1972) pourrait constituer un avantage zootechnique, bien qu'une confirmation sur un plus grand nombre de descendants de mâles porteurs du chimérisme $\mathrm{XX} / \mathrm{XY}$, soit nécessaire.

Reçu pour publication en août 1975.

\section{REMERCIEMENTS}

Nous remercions le centre d'insémination artificielle (Ente Lombardo per il Potenziamento zootecnico) de Zorlesco (Milan) pour la collaboration accordée, qui nous a permis de réaliser cette étude.

\section{SUMMARY}

\section{A FIRST CYTOGENETIC STUDY IN AN ITALIAN A.I. CENTRE}

A study of 39 bulls from an Italian station for artificial insemination has been made. No chromosomal abnormality has been found. One Holstein-Friesian bull was found to have an $\mathrm{XX} / \mathrm{XY}$ chimaerism. He had $52,38 \mathrm{p}$. 1 oo female cells, a low fertility in comparison with the mean fertility of this station and a deviation in the sex-ratio in favour of females.

\section{RIASSUNTO}

\section{UN PRIMO STUDIO CITOGENETICO}

\section{IN UN CENTRO ITALIANO DI FECONDAZIONE ARTIFICIALE}

Uno studio citogenetico è stato svolto su 39 tori di un Centro di Fecondazione artificiale italiano. Non sono state trovate anomalie cromosomiche nè nel numero nè nella struttura, mentre è stato possibile mettere in evidenza un chimerismo $\mathrm{XX} / \mathrm{XY}$ in un toro di razza Frisona pezzata nera di ceppo U.S. A. Questo soggetto presentava una percentuale del $52,3^{8} \mathrm{p}$. roo di cellule femminili, una fertilità ridotta in rapporto alla media del centro ed una sex-ratio a favore del sesso femminile. 


\section{RÉFÉRENCES BIBLIOGRAPHIQUES}

Basrur P. K., Gilman J. P. W., McSherry B. J., I964. Cytological observations on a bovine lymphosarcoma. Nature, 201, 368.

Bouters R., Vandeplassche H., r964. The male sex chromosome as a possible cause of the development of freemartinism in cattle. Vlaam. Diergences bunding Tijdschr, 38, 229-241.

Bruere A. N., Chapman H. M., 1973. Autosomal translocation in two exotic breeds of cattle in New-Zealand. Vet. Rec., 92, 615.

De Grouchy J., Roubin M., Passage E., r964. Microtechnique pour l'étude des chromosomes humains à partir d'une culture de leucocytes sanguins. Ann. Génét., 7, 45.

Dunn H. O., Kenney R. M., Stone W. H., Bendel S., r 1968 . Cytogenetic and reproductive studies of $\mathrm{XX} / \mathrm{XY}$ chimeric twin bulls. VIe Cong. Anim. Reprod., Paris, 1968, 2, 877-879.

Dunn H. O., Johnson R. H., 1972. A 6r, XY cell line in a calf with extreme brachygnathia. $J$. Dairy Sci., 55, 524-526.

Fechheimer N. S., Herschler M. S., Gilmore L. O., r963. Sex chromosome mosaicisme in unlike sexed cattle twins. XIth Int. Cong. Genet. The Hague, 1963, 1, 265. (Pergamon Press).

FechHeimer N. S., 1973. A cytogenetic survey of young bulls in the U. S. A. I'et. Rec., 98, 535-536.

Gustavsson I., Fraccaro M., Tiepolo L., Lindsten J., I968. Presumptive X-autosome translocation in a cow. Preferential inactivation of the normal X chromosome. Nature, 218, 183.

Gustavsson I., I969. Cytogenetics, distribution and phenotypic effects of a translocation in swedish cattle. Hereditas, 63, 68-169.

Hansen K. M., 1969. Bovine tandem fusion and infertility. Hereditas, 63, 453.

Herschler M. S., Fechieimer N. S., r966. Centric fusion of chromosomes in a set of bovine triplets. Cytogenetics, 5, 307-312.

Herzog A., HöhN H., I968. Autosomale trisomie bei einem kalt mit Brachygnatie inferior und Ascites Congenitus. Dtsch. Tierärtztl. Wochenschr., 75, 604 .

Herzog A., HönN H., I97x. Autosomale trisomie bei der letalen Brachygnathie des Rindes. Cytogenetics, 10, 347-355.

Keller K., TANDleR J., I9I6. The behaviour of the chorion in twin pregnancies of cattle. Wien. Tieräratl. Monatsschr., 8, 5 I3.

LILlie F. R., I9I6. The theory of the Freemartin. Science, 43, 6II.

Lillie F. R., rgr7. The freemartin; a study of the action of sex hormones in the foetal life of cattle. J. Exp. Zool., 23, 37 1-452.

LoJDA L., I972. Chromosomal chimerisme of the sire as a cause of the shift in sex ratio in his offspring. $V I I^{\mathrm{e}}$ Congr. int. reprod. anim. insémin. artif., Munich, juin 1972, $1 \mathrm{III-III3.}$

Mori M., SaSaki M., Ishikawa T., Kawata K., 1969. Autosomal trisomy in a malformed new born calf. Proc. Japan Acad., 45, 955.

Ohno S., Trussillo J. M., Stenius C., Christian L. C., Teplitz R. L., ig62. Possible germ cell chimeras among newborn dizygotic twin calves (Bos taurus). Cytogenetics, 1, 258.

Pollock D. L., 1972. A chromosome abnormality in Friesan cattle in Great Britain. I'et. Rec., 90, 309.

Pollock D. L., 1974. Chromosome studies in artificial insemination sires in Great Britain. Vet. Rec., 95, 266-267.

Rıeck G. W., Höhn H., Herzog A., I97o. $\mathrm{X}$ trisomie beim Rind mit Anzeichen familiärer Disposition für Meioses-törungen. Cytogenetics, 8, 40r.

Short R. V., r969. An introduction to some of the problems of intersexuality. J. Reprod. Fert. suppl., 7, $\mathrm{r}-8$.

Stafford M. Y., I972. The fertility of bull born co-twin to heifer. Vet. Rec., 80, I46-I48. 